Department of Biology and Pharmaceutical Botany, Ludwik Rydygier Collegium Medicum in Bydgoszcz, Nicolaus Copernicus University in Toruń, SkłodowskiejCurie 9, 85-094 Bydgoszcz, Poland, tzaluski@cm.umk.pl

\title{
VEGETATION TRANSFORMATIONS OF KUJAWY-POMERANIA REGION IN THE LAST TWENTY YEARS PERIOD
}

\begin{abstract}
This paper contents dynamic tendencies analysis results of KujawyPomerania region (N Poland) for a 20-year time period. Acreage changes as well as structure and species composition transformations of forest and shrub vegetation (associations) and non-forest vegetation (alliances) were evaluated. Main factors influencing on regressive and progressive changes were set out. Analysis was relied on results of geobotanical research and observations of selected objects. Regressive changes were noted for i.a. thermophilous oak forests, dry and fresh coniferous forests, aquatic, halophytic, pasture vegetation, vegetation of Molinion and Cnidion meadows, heaths and xerothermic grasslands. Whereas progressive were observed in case of mixed coniferous forests, mesophytic shrubs, rush and tall herbs vegetation, fresh meadows and some ruderal communities.
\end{abstract}

Key words: causes of transformations, ecological processes, plant communities, vegetation dynamics

\section{INTRODUCTION}

Existence of plant cover dynamic tendencies is well-known. Plant species and plant communities reveal dynamics under the influence of various natural factors and diverse, variables in space and time, anthropogenic factors (MICHALIK 1974; OLACZEK 1976, SENDEK 1981; KUCHARSKI 1999; JUTRZENKA-TRZEBIATOWSKI 
1999; FALIŃSKA 2004). Researchers' interests in this matter focus both on species population dynamics (taxons' threats) as well as on transformations of ecosystems and plant communities in a broad sense.

Concluding basis about all vegetation changes constitutes among others a possibility of comparison of current state with the past state. Since in the last few decades in Poland intense phytosociological research were carried out, analyses of vegetation transformations are currently undertaken more often. It concerns both different types of plant communities as well as different regions of our country (KORNAŚ 1987; MiCHALIK 1990a, b; KoRNAŚ, DUBIEL 1991; ZARZYCKI, KORZENIAK 1992; JAKUBOWSKA-GABARA 1993; KUROWSKI 1993; BARABASZ 1997; ZAŁUSKI 2002; SugIER, CZARnecka 2004; BODZIARCZYK, DraJEwICZ 2007; KaźMierCZaKowa, GrodzIŃSKa 2007; Kurowski, Michalska-HeJduk 2007; MichalSKA-HEJDUK 2007; WróBel 2007; ZARZYCKI, KAŹMIERCZAKOWA 2007; MATUSZKIEWICZ 2007a, DURAK 2009; GRYNIA et al. 2009).

This paper is an attempt of knowledge balancing on the subject of natural and anthropogenic transformations of different vegetation types in KujawyPomerania region. It is an original point of view on plant cover of this region because evaluations thereof dynamic tendencies rely not only on documentation but also on different objects' observations. For a clearer image of vegetation changes a relatively short period of time was adopted - a period of the last 20 years. At the same time, it is a period of other than previously performed forms of nature use resulting from different economic reality.

\section{MATERIAL AND METHODS}

An analysis of dynamic tendencies of vegetation was carried our for KujawyPomerania province ( $\mathrm{N}$ Poland) including some of its adjacent areas. It is a nonhomogenous area in physicogeographical terms, constituting a part of few macroregions - South Pomeranian Lakeland, Wielkopolska Lakeland, ToruńEberswalde Glacial Valley, Lower Vistula Valley and Chełmno-Dobrzyń Lakeland (KONDRACKI 2000). Vegetation transformations analyses were performed for the last twenty years period. 
Both, acreage changes and degree of structure species composition transformation of respected vegetation types were evaluated. Main factors influencing on regressive and progressive changes were set out. As a basic unit of forest and shrub vegetation unit an association was adopted, whereas of non-forest vegetation - an alliance. A setout of values and causes of changes was presented in tables.

Syntaxonomical classification of forest, meadow and partially ruderal vegetation was adopted according to MATUSZKIEWICZ (2005), spring vegetation according to HINTERLANG (1992), whereas other non-forest plant communities according to BRZEG and WOJTERSKA (2001).

The analysis was relied on results of author's research and other authors' research and also on observations of selected, previously geobotanically identified objects. Numerous published and unpublished papers concerning both forest (i.a. KĘPCZYŃSKI, ZIELSKI 1976; ZIELSKI 1978; BERNDT, CEYNOWA-GIEŁDON 1988; ZIELONY 1988; KęPCZYŃSKI, ZAŁUSKi 1991; CyZMAN, REJEWSKI 1992; BIAŁY, ZAŁUSKI 1994; ZAŁUSKI, GAWENDA 1999; JAGODZIŃSKI, MACIEJEWSKARutKowska 2005, 2008; PASZEK 2005; MATUSZKIEWICZ 2007b; CyZMAN, KANNENBERG 2008; CYZMAN 2009) and non-forest (i.a. WILKOŃ-MiCHALSKA 1970; KęPCZYŃSKI, ZAŁUSKI 1977, 1988; NORYŚKIEWICZ 1978; SAMOSIEJ 1987; KęPCZYŃSKI， CEYNOWA-GIEŁDON 1988; KRASICKA-KORCZYŃSKA 1996; MARCYSIAK 1999; ZAŁUSKI et al. 2005, 2008; EJANKOWSKI, KUNZ 2006; KRASICKAKORCZYŃSKA et al. 2008; RATYŃSKA 2008; WALDON, RAPACKA-GACKOWSKA 2010; KUNZ, NiENARTOWICZ 2010; NIENARTOWICZ et al. 2010) vegetation changes were used. For each analyzed syntaxonomical unit documented and observed changes in at least 5 objects were taken into account. A detailed documentation of performed analyses and evaluations is located in Department of Biology and Pharmaceutical Botany, Ludwik Rydygier Collegium Medicum in Bydgoszcz, Nicolaus Copernicus University in Torun. 


\section{RESULTS AND DISCUSSION}

Dynamic tendencies analysis of deciduous forests (Tab. 1) revealed that few plant communities from different phytosociological classes undergo distinct transformations.

Forest plant communities revealing regressive tendencies comprise eutrophic swamp forests Ribeso nigri-Alnetum, riparian poplar forests Populetum albae, higrophilous elm forests (Ficario-Ulmetum minoris, Violo odoratae-Ulmetum), oaklinden-hornbeam forests (Tilio-Carpinetum, Galio-Carpinetum) and thermophilous oak forests Potentillo albae-Quercetum (Tab. 1). About regressive tendencies of Ribeso nigri-Alnetum, including transformations of this plant community towards wet alder riparian forests Fraxino-Alnetum, decides mainly lowering from many years ground water level (cf. CYZMAN 2009). Riparian poplar forests Populetum albae have larger share of geographically alien species (i.a. Acer negundo, Solidago gigantea) and reveal transformations towards higrophilous elm forests FicarioUlmetum minoris. Higrophilous elm forests (Ficario-Ulmetum minoris, Violo odoratae-Ulmetum) reveal regression as a result of inundations which leads to changes towards oak-linden-hornbeam forest (cf. JAGODZIŃSKI, MACIEJEWSKARutKowsKa 2005, 2008; CYZMAN, KANNENBERG 2008). Oak-linden-hornbeam forests (Tilio-Carpinetum, Galio-Carpinetum) undergo degeneration (pinetization) as a result of coniferous trees introduction, which is a well-known process (OLACZEK 1974; ZIELSKI 1978; ZIELONY 1988; CYZMAN 1991). They also reveal gradual transformations as a result of abundant development of different deciduous trees or shrubs, which is a cause of strong shadowing of herb layer. Furthermore, they are exposed to danger of kenophytes' penetration (e.g. Impatiens parviflora). Heliophilous and thermophilous oak forests Potentillo albae-Quercetum, which can be acknowledged as the most endangered forest community (cf. JAKUBOWSKAGABARA 1993), undergo analogical transformations. Changes occurring in their phytocoenoses are very clear and follow generally in a quick pace. 
Table 1. Transformations of deciduous forest vegetation. Changes of area: $-1-$ small decrease; -2 - distinct decrease; 0 - lack of changes or small changes; +1 - small increase; +2 - distinct increase; ? - disputable assessment. Changes of structure and species composition: $\downarrow$ - small changes due to regression/degeneration; $\downarrow \downarrow$ - distinct changes due to regression/degeneration; $\leftrightarrow$ - lack of changes or small changes; $\uparrow ~-$ small changes due to succession/regeneration; $\uparrow \uparrow$ - distinct changes due to succession/regeneration; ? - disputable assessment. Main causes: aff - afforestation; asexp - alien species expansion; asint - alien species introduction; bua - built-up areas; csint - coniferous species introduction; eh - eutrophication of habitats; fe fishpond establishment; ff - forest felling; fl - floods; freg - forest regeneration; gwld - ground water level decrease; nrp - natural regression processes; nsp - natural succession processes; saf - shadowing by adjacent forests; ssuccu - secondary succession due to cessation of use; ssw - secondary swamping; tbulb - technical building up of lake banks; tburb - technical building up of river banks; tm - treestand monotypization; tr - trampling; tru - tourist and recreation use.

\begin{tabular}{|c|c|c|c|c|}
\hline Syntaxonomic units & 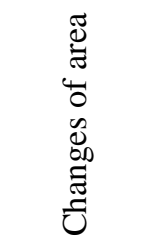 & 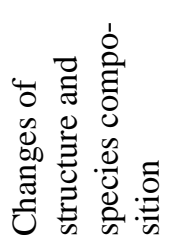 & 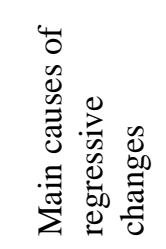 & 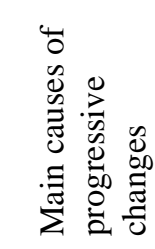 \\
\hline \multicolumn{5}{|c|}{ Alnetea glutinosae Br.-Bl. et R. Tx. 1943; Alnion glutinosae (Malc. 1929) Meij. Drees. 1936} \\
\hline $\begin{array}{l}\text { Ribeso nigri-Alnetum Sol.-Górn. (1975) } \\
1987\end{array}$ & $-2,0,+1$ & $\downarrow \downarrow \leftrightarrow \uparrow$ & $\begin{array}{l}\text { bua, fe, ff, } \\
\text { gwld, nrp }\end{array}$ & $\begin{array}{l}\text { freg, nsp, } \\
\text { ssuccu, } \\
\text { ssw }\end{array}$ \\
\hline $\begin{array}{l}\text { Sphagno squarrosi-Alnetum Sol-Górn. } \\
\text { (1975) } 1987\end{array}$ & $-1,0,+1$ & $\downarrow \leftrightarrow \uparrow$ & eh, gwld & freg, nsp \\
\hline $\begin{array}{l}\text { Cardamino-Alnetum (Meij. Drees. 1936) } \\
\text { Pass. } 1968\end{array}$ & $-1,0$ & $\leftrightarrow$ & fe & \\
\hline $\begin{array}{l}\text { Thelypterido-Betuletum pubescentis } \\
\text { Czerw. } 1972\end{array}$ & $-1,0,+1$ & $\downarrow \leftrightarrow \uparrow$ & $\begin{array}{l}\text { eh, fe, } \\
\text { gwld }\end{array}$ & $\begin{array}{l}\text { freg, nsp, } \\
\text { ssuccu, } \\
\text { ssw }\end{array}$ \\
\hline \multicolumn{5}{|c|}{ Salicetea purpureae Moor 1958; Salicion albae R. Tx. 1955} \\
\hline Salicetum albo-fragilis R. Tx. 1955 & $-1,0,+1$ & $\downarrow \leftrightarrow \uparrow ?$ & $\begin{array}{l}\text { asexp, } \\
\text { gwld, nrp, } \\
\text { tburb }\end{array}$ & $\begin{array}{l}\mathrm{fl}, \mathrm{nsp} \\
\text { ssuccu, } \\
\text { ssw }\end{array}$ \\
\hline Populetum albae Br.-B1. 1931 & $-1,0,+1$ & $\downarrow \downarrow \leftrightarrow \uparrow$ & $\begin{array}{l}\text { asexp, } \\
\text { asint, } \\
\text { gwld, nrp, } \\
\text { tm }\end{array}$ & $\begin{array}{l}\text { fl, freg, } \\
\text { nsp }\end{array}$ \\
\hline
\end{tabular}

Querco-Fagetea Br.-Bl. et Vlieg. 1937 ; Alno-Ulmion Br.-Bl. et R. Tx. 1943

\begin{tabular}{|c|c|c|c|c|}
\hline Fraxino-Alnetum W. Mat. 1952 & $-1,0,+2$ & $\downarrow \leftrightarrow \uparrow \uparrow$ & $\begin{array}{l}\text { asexp, bua, } \\
\text { fe, ff, } \\
\text { gwld, ssw, } \\
\text { tbulb, tm, } \\
\text { tru }\end{array}$ & $\begin{array}{l}\text { aff, freg, } \\
\text { nsp, ssuccu }\end{array}$ \\
\hline $\begin{array}{l}\text { Carici remotae-Fraxinetum W. Koch } 1926 \\
\text { ex Faber } 1936\end{array}$ & $-1,0$ & $\leftrightarrow$ & fe & \\
\hline Astrantio-Fraxinetum Oberd. 1953 & $0 ?$ & $\leftrightarrow ?$ & & \\
\hline
\end{tabular}


Table 1. (Continued)

\begin{tabular}{|c|c|c|c|c|}
\hline $\begin{array}{l}\text { Ficario-Ulmetum minoris Knapp } 1942 \text { em. } \\
\text { J. Mat. } 1976 \\
\text { Violo odoratae-Ulmetum minoris } \\
\text { (Weevers 1940) Doing } 1962\end{array}$ & $-1,0,+1$ & $\begin{array}{l}\downarrow \downarrow \leftrightarrow \uparrow \\
\downarrow \downarrow \leftrightarrow \uparrow\end{array}$ & $\begin{array}{l}\text { csint, nrp, } \\
\text { tm } \\
\text { asint, csint, } \\
\text { nrp, tm }\end{array}$ & $\begin{array}{l}\text { aff, freg, } \\
\text { nsp } \\
\text { freg, nsp }\end{array}$ \\
\hline \multicolumn{5}{|c|}{ Carpinion betuli Issler 1931 em. Oberd. 1953} \\
\hline $\begin{array}{l}\text { Tilio cordatae-Carpinetum betuli Tracz. } \\
1962\end{array}$ & $-1,0,+1$ & $\downarrow \downarrow \leftrightarrow \uparrow$ & \multirow{3}{*}{$\begin{array}{l}\text { asexp, } \\
\text { asint, csint, } \\
\text { nrp, tru } \\
\text { asexp, } \\
\text { asint, csint, } \\
\text { nrp, tru } \\
\text { asexp, tr }\end{array}$} & $\begin{array}{l}\text { aff, freg, } \\
\text { nsp }\end{array}$ \\
\hline $\begin{array}{l}\text { Galio sylvatici-Carpinetum betuli Oberd. } \\
1957\end{array}$ & $-1,0,+1$ & $\downarrow \downarrow \leftrightarrow \uparrow$ & & $\begin{array}{l}\text { aff, freg, } \\
\text { nsp }\end{array}$ \\
\hline $\begin{array}{l}\text { Acer platanoides-Tilia cordata Jutrz.- } \\
\text { Trzeb. } 1995\end{array}$ & 0 & $\downarrow \leftrightarrow$ & & \\
\hline \multicolumn{5}{|l|}{ Fagion sylvaticae R. Tx. et Diem. 1936} \\
\hline $\begin{array}{l}\text { Luzulo pilosae-Fagetum W. Mat. et A. } \\
\text { Mat. } 1973\end{array}$ & $0,+1$ & $\downarrow \leftrightarrow \uparrow$ & \multirow{2}{*}{$\begin{array}{l}\text { csint, saf, } \\
\text { tru } \\
\text { csint, tru }\end{array}$} & freg, nsp \\
\hline $\begin{array}{l}\text { Galio odorati-Fagetum Rübel } 1930 \text { ex } \\
\text { Sougnez et Thill } 1959\end{array}$ & $0,+1$ & $\leftrightarrow \uparrow$ & & freg, nsp \\
\hline \multicolumn{5}{|c|}{ Potentillo albae-Quercion petraeae Zól. et Jakucs n.nov. Jakucs 1967} \\
\hline $\begin{array}{l}\text { Potentillo albae-Quercetum petraeae Libb. } \\
1933 \text { n.inv. }\end{array}$ & $-2,0$ & $\downarrow \downarrow \leftrightarrow \uparrow$ & $\begin{array}{l}\text { asexp, } \\
\text { asint, csint, } \\
\text { nrp, saf, tm }\end{array}$ & $\begin{array}{l}\text { ff, freg, } \\
\text { nsp }\end{array}$ \\
\hline
\end{tabular}

Whereas into group of forest plant communities which reveal progressive tendencies alder riparian forests Fraxino-Alnetum and beech forests can be reckoned (Tab. 1). Communities of a wet alder riparian forests Fraxino-Alnetum character develop in areas of drained swamp forests Ribeso nigri-Alnetum and as a consequence of spontaneous or conscious afforestation of wet meadows. Whereas gradual yet more distinct share increase of beech forests (Luzulo pilosae-Fagetum, Galio odorati-Fagetum) in some forest complexes results from natural and anthropogenic share increase of beech Fagus sylvatica in deciduous and mixed coniferous forests' treestands.

Other deciduous forest associations (Tab. 1) are relatively stable on account of occupied area as well as structure and species composition, while undergoing changes in many observed phytocoenoses are not distinct. Particularly stable vegetation units are some plant communities of wet forests, mainly spring forests (Cardamino-Alnetum, Carici remotae-Fraxinetum, Astrantio-Fraxinetum) and slope maple-linden forest Acer platanoides-Tilia cordata. About stable character of this communities decide both natural stability of habitats and low availability for human management. 
A setout of dynamic tendencies of coniferous and mixed coniferous forests vegetation (Tab. 2) depicts a specific regularity. It is and area shrinking and disappearing of individual features of phytocoenoses of oligotrophic dry and fresh pine coniferous forests (Cladonio-Pinetum, Peucedano-Pinetum, LeucobryoPinetum), while progressive tendencies for mixed coniferous forests (Querco roboris-Pinetum, Serratulo-Pinetum), typical for mesotrophic habitats. This direction of changes was documented in examined area (KĘPCZYŃSKI, ZAŁUSKI 1991; PASZEK 2005) and in other regions of Poland (MATUSZKIEWICZ 2007c). Changes mentioned above are generated both by natural factors, i.e. regeneration and succession processes on mesotrophic habitats, as well as supported by anthropogenic actions, mainly by preferring deciduous trees (Quercus spp.) in accordance with principles of contemporary forest management. Very clear disappearing tendencies of acid oak forests (Calamagrostio arundinaceaeQuercetum petraeae), barely noted in examined region should be underlined.

Analysis of changes of shrub and clearing vegetation (Tab. 3) revealed generally high variability of dynamic tendencies of respective phytocoenoses. Few plant associations reveal progressive tendencies (Salicetum pentandro-cinereae, Euonymo-Prunetum spinosae, Aegopodio-Sambucetum nigrae, Agrostio-Populetum tremulae, Rubo plicati-Sarothamnetum), which is an effect of extensive forms of use and lack of use of meadows and grasslands. Disappearing communities - Betuletum humilis and Prunetum fruticosae, of which dominants are strongly endangered species were also noted (cf. ZARZYCKI, SZELĄG 2006).

Regressive tendencies of many groups of aquatic vegetations were demonstrated (Tab. 4), which is conditioned by gradual natural or anthropogenic eutrophication of water bodies and thereby a water transparency decrease (cf. REJEWSKI 1981). Disappearing are also silt-covered ground communities of IsoëtoJuncetea bufonii class, which is mostly connected with developing and edge regulation of small, mid-field water bodies and lakes. 
Table 2. Transformations of coniferous and mixed forest vegetation. Changes of area: explanations in Table 1. Changes of structure and species composition: explanations in Table 1. Main causes: aff - afforestation; asexp - alien species expansion; asint - alien species introduction; csint - coniferous species introduction; eh - eutrophication of habitats; ff - forest felling; freg - forest regeneration; gwld ground water level decrease; nrp - natural regression processes; nsp - natural succession processes; saf - shadowing by adjacent forests; tm - treestand monotypization; tru - tourist and recreation use; tu - turfing.

\begin{tabular}{|c|c|c|c|c|}
\hline Syntaxonomic units & 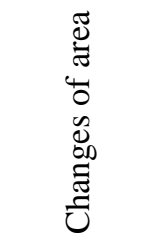 & 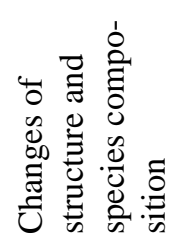 & 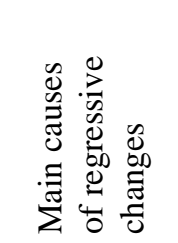 & 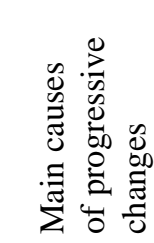 \\
\hline \multicolumn{5}{|c|}{ Quercetea robori-petraeae Br.-Bl. et R. Tx. 1943 ; Quercion robori-petraeae Br.-B1. 1932} \\
\hline $\begin{array}{l}\text { Calamagrostio arundinaceae-Quercetum } \\
\text { petraeae (Hartm. 1934) Scam. et Pass. } \\
1959\end{array}$ & -2 & $\downarrow \downarrow ?$ & $\begin{array}{l}\text { csint, nrp, } \\
\text { tm }\end{array}$ & \\
\hline \multicolumn{5}{|c|}{ Vaccinio-Piceetea Br.-Bl. in Br.-Bl. et al. 1939; Dicrano-Pinion (Libb. 1933) W. Mat. 1962} \\
\hline Cladonio-Pinetum Juraszek 1927 & $-2,0,+1$ & $\downarrow \downarrow \leftrightarrow \uparrow$ & \multirow{3}{*}{$\begin{array}{l}\text { asint, eh, } \\
\text { nrp, saf } \\
\text { asexp, } \\
\text { asint, eh, } \\
\text { ff, nrp, saf, } \\
\text { tru, tu } \\
\text { asexp, } \\
\text { asint, eh, } \\
\text { ff, nrp, tru, } \\
\text { tu }\end{array}$} & $\begin{array}{l}\text { aff, ff, } \\
\text { freg, nsp }\end{array}$ \\
\hline $\begin{array}{l}\text { Peucedano-Pinetum (W. Mat. 1962) W. } \\
\text { Mat. et J. Mat. } 1973\end{array}$ & $-2,0,+1$ & $\downarrow \downarrow \leftrightarrow \uparrow$ & & $\begin{array}{l}\text { aff, freg, } \\
\text { nsp }\end{array}$ \\
\hline $\begin{array}{l}\text { Leucobryo-Pinetum (W. Mat. 1962) W. } \\
\text { Mat. et J. Mat. } 1973\end{array}$ & $-2,0,+1$ & $\downarrow \downarrow \leftrightarrow \uparrow$ & & $\begin{array}{l}\text { aff, freg, } \\
\text { nsp }\end{array}$ \\
\hline $\begin{array}{l}\text { Molinio (caeruleae)-Pinetum W. Mat et J. } \\
\text { Mat. } 1973\end{array}$ & $0 ?$ & $\downarrow \leftrightarrow ?$ & $\begin{array}{l}\text { asexp, } \\
\text { asint, tu }\end{array}$ & \\
\hline $\begin{array}{l}\text { Querco roboris-Pinetum (W. Mat 1981) J. } \\
\text { Mat. } 1988\end{array}$ & $-1,0,+2$ & $\downarrow \leftrightarrow \uparrow \uparrow$ & $\begin{array}{l}\text { asexp, } \\
\text { asint, csint, } \\
\text { nrp, tm, } \\
\text { tru, tu }\end{array}$ & $\begin{array}{l}\text { aff, freg, } \\
\text { nsp }\end{array}$ \\
\hline $\begin{array}{l}\text { Serratulo-Pinetum (W. Mat 1981) J. Mat. } \\
1988\end{array}$ & $-1,0,+2$ & $\downarrow \leftrightarrow \uparrow$ & $\begin{array}{l}\text { asexp, } \\
\text { asint, csint, } \\
\text { nrp, tm, tru }\end{array}$ & freg, nsp \\
\hline $\begin{array}{l}\text { Vaccinio uliginosi-Betuletum pubescentis } \\
\text { Libb.1933 }\end{array}$ & $-1,0,+1$ & $\downarrow \leftrightarrow \uparrow$ & $\begin{array}{l}\text { asexp, } \\
\text { csint, } \\
\text { gwld, nrp }\end{array}$ & freg, nsp \\
\hline Vaccinio uliginosi-Pinetum Kleist 1929 & $0,+1$ & $\downarrow \leftrightarrow \uparrow$ & eh, gwld & $\mathrm{nsp}$ \\
\hline \multicolumn{5}{|l|}{ Vaccinio-Piceenion Oberd. 1957} \\
\hline $\begin{array}{l}\text { Querco-Piceetum (W. Mat. 1952) W. Mat. } \\
\text { et Pol. } 1955\end{array}$ & $0 ?$ & $\downarrow \leftrightarrow \uparrow$ & csint, nrp & aff, nsp \\
\hline
\end{tabular}


Table 3. Transformations of shrub and clearing vegetation. Changes of area: see explanations in Table 1. Changes of structure and species composition: see explanations in Table 1. Main causes: asexp - alien species expansion; bua - built-up areas; cfag - conversion into farmlands or allotment gardens; eg - establishment of greenway; eh - eutrophication of habitats; fe - fishpond establishment; ff - forest felling; fl - floods; freg - forest regeneration; gwld - ground water level decrease; le lawn establishment; lr - levelling of roadsides; nrp - natural regression processes; nsp - natural succession processes; $\mathrm{p}$ - plantings; saf - shadowing by adjacent forests; ssuccu - secondary succession due to cessation of use; ssw - secondary swamping; tburb - technical building up of river banks; we - wastelands elimination.

\begin{tabular}{|c|c|c|c|c|}
\hline Syntaxonomic units & 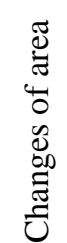 & 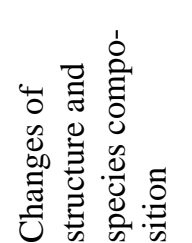 & 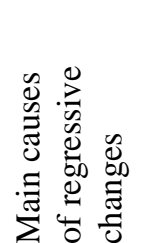 & 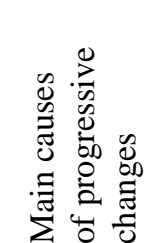 \\
\hline
\end{tabular}

Alnetea glutinosae Br.-Bl. et R. Tx. 1943; Salicion cinereae (Malc. 1929) Meij. Drees. 1936

Salicetum pentandro-cinereae (Almq. 1929) $\quad-1,0,+2 \quad \downarrow \leftrightarrow \uparrow \quad$ asexp, fe, $\quad n s p$, ssuccu

Pass. 1961

Salicetum auritae Jonas $1935 \mathrm{em}$. Oberd. $\quad-1,0,+1 \quad \downarrow \leftrightarrow \uparrow \quad$ eh, gwld, $\quad$ nsp

1964

nrp

Betuletum humilis Steffen $1931 \quad-2,0 \quad \downarrow \downarrow \leftrightarrow \quad$ gwld, nrp

Salicetea purpureae Moor 1958; Salicion albae R. Tx. 1955

Salicetum triandro-viminalis Lohm. $1952 \quad-1,0,+2 \quad \downarrow \leftrightarrow \uparrow \quad$ asexp, nrp, $\quad$ fl, nsp tburb

Rhamno-Prunetea Rivas-Goday et Carb. 1961 ex R. Tx. 1962; Carpino-Prunion spinosae R. Tx. 1952 em. Weber 1974

Euonymo-Prunetum spinosae (Hueck 1931) $\quad-1,0,+2 \quad \downarrow \leftrightarrow \uparrow \quad$ lr, nrp, we $\quad$ nsp, ssuccu

Pass. in Pass. et Hofm. 1968

Euonymo-Coryletum Pass. in Pass. et $\quad-1,+1 \quad \downarrow \leftrightarrow \uparrow \quad$ 1r, nrp $\quad$ nsp, ssuccu

Hofm. 1968

Berberidion Br.-B1. 1950 ex R. Tx. 1952

Pruno-Ligustretum R. Tx. 1952 nom.inv. $\quad-1,0,+1 \quad \downarrow \leftrightarrow \uparrow \quad$ nrp, we $\quad$ nsp, ssuccu

Prunion fruticosae R. Tx. 1952

Prunetum fruticosae Dziubałtowski $1926 \quad-1 \quad$-1 $\quad$ r $\quad$ nrp

Arctio-Sambucion nigrae Doing 1962

Aegopodio-Sambucetum nigrae Doing 1962

em. M. Wojt. 1990

Chelidonio-Robinietum Jurko 1963

$-1,0,+2 \quad \downarrow \leftrightarrow \uparrow \quad$ asexp, bua,

ssuccu

$-1,0,+1 \quad \downarrow \leftrightarrow \uparrow \quad$ bua, eg, le, p, asexp

Agrostio capillaris-Frangulion Pass. in Pass. et Hofm. 1968 em. Brzeg et M. Wojt. 2001

\begin{tabular}{|c|c|c|c|c|}
\hline $\begin{array}{l}\text { Molinio-Franguletum Pass. in Pass. et } \\
\text { Hofm. } 1968 \text { em. Brzeg et M. Wojt. } 2001\end{array}$ & $-1,0,+1$ & $\leftrightarrow \uparrow ?$ & nrp, we & nsp, ssuccu \\
\hline $\begin{array}{l}\text { Agrostio-Populetum tremulae Pass. in } \\
\text { Pass. et Hofm. } 1968\end{array}$ & $-1,0,+2$ & $\downarrow \leftrightarrow \uparrow \uparrow$ & $\begin{array}{l}\text { bua, cfag, } \\
\text { we }\end{array}$ & nsp, ssuccu \\
\hline Rubo plicati-Sarothamnetum Weber 1977 & $-1,0,+2$ & $\downarrow \leftrightarrow \uparrow$ & $\begin{array}{l}\text { cfag, freg, } \\
\text { lr, nrp, saf, } \\
\text { we }\end{array}$ & $\begin{array}{l}\text { ff, nsp, } \\
\text { ssuccu }\end{array}$ \\
\hline
\end{tabular}


Table 3. (Continued)

\begin{tabular}{|c|c|c|c|c|}
\hline ass. Rubus plicatus & $-1,0,+1$ & $\downarrow \leftrightarrow \uparrow$ & $\begin{array}{l}\text { freg, lr, } \\
\text { nrp, saf, } \\
\text { we }\end{array}$ & $\begin{array}{l}\text { ff, nsp, } \\
\text { ssuccu }\end{array}$ \\
\hline \multicolumn{5}{|c|}{$\begin{array}{l}\text { Epilobietea angustifolii R. Tx. et Prsg 1950; Sambuco-Salicion capreae R. Tx. et Neum } \\
\text { R. Tx. } 1950 \text { ex Oberd. } 1957\end{array}$} \\
\hline Salicetum capreae Schreier 1955 & $-1,0,+1 ?$ & $\downarrow \leftrightarrow \uparrow ?$ & $\begin{array}{l}\text { freg, lr, } \\
\text { nrp, saf }\end{array}$ & ff, nsp \\
\hline $\begin{array}{l}\text { Rubetum idaei Malinowski et } \\
\text { Dziubałtowski } 1914 \text { em. Oberd. } 1973\end{array}$ & $-1,+1$ & $\downarrow \leftrightarrow \uparrow$ & $\begin{array}{l}\text { freg, lr, } \\
\text { nrp, saf }\end{array}$ & $\mathrm{ff}, \mathrm{nsp}$ \\
\hline $\begin{array}{l}\text { Sambucetum racemosae (Noirf. 1949) } \\
\text { Oberd. } 1973\end{array}$ & $-1,+1 ?$ & $\downarrow \leftrightarrow \uparrow ?$ & $\begin{array}{l}\text { freg, lr, } \\
\text { nrp, saf }\end{array}$ & ff, nsp \\
\hline $\begin{array}{l}\text { Carici piluliferae-Epilobion angustifolii } \mathrm{R} \text {. } \\
\text { Tx. } 1950\end{array}$ & $-1,0,+1$ & $\downarrow \leftrightarrow \uparrow$ & freg, lr, nrp & ff, nsp \\
\hline
\end{tabular}

Distinct progressive tendencies are noted for different rush and tall sedge vegetation (all. Phragmition, Magnocaricion, Phalaridion; Tab. 4). On water bodies' edges it is usually an effect of shallowing and eutrophication, whereas in grasslands complexes - an effect of cessation of use and secondary swamping. Clearly spreading plant communities constitute i.a. Phragmitetum australis and Phalaridetum arundinaceae.

Relatively stable are spring communities (Tab. 4). Pioneer halophytic communities (all. Salicornion ramosissimae) and adjacent therophytes (all. Bidention tripartitae, Chenopodion glauci), in turn, reveal irregular appearance dependent on water level and soil humidity fluctuations.

Specific plant cover dynamics is observed on peatlands (Tab. 4). More or less boggy peat communities (Rhynchosporion albae, Caricion lasiocarpae, Sphagnion magellanici) are currently relatively stable or undergo gradual regression as a result of shrub and forest vegetation succession. The most vulnerable on unfavourable changes (eutrophication and then shrub and forest succession) is however typical to fen vegetation (Caricion nigrae, $C$. davallianae, partially $C$. lasiocarpae), especially moss sedge fen (cf. HeRвichowa, WoŁejKo 2004). The least vulnerable are then transformations of phytocoenoses of oligotrophic and strongly bogged habitats. 
Table 4. Transformations of aquatic, spring, coastal and peat vegetation. Changes of area: explanations in Table 1. Changes of structure and species composition: explanations in Table 1. Main causes: aff - afforestation; asexp - alien species expansion; del - developing of edge lakes; eh - eutrophication of habitats; fe fishpond establishment; fl - floods; gwld - ground water level decrease; lr - levelling of roadsides; lssw - limitation of supply with saline water; nrp - natural regression processes; nsp - natural succession processes; saf - shadowing by adjacent forests; ssuccu - secondary succession due to cessation of use; ssw - secondary swamping; swp - surface water pollution; tbulb - technical building up of lake banks; tburb technical building up of river banks; tr - trampling; tru - tourist and recreation use; tu - turfing; we - wastelands elimination; wld - water level decrease.

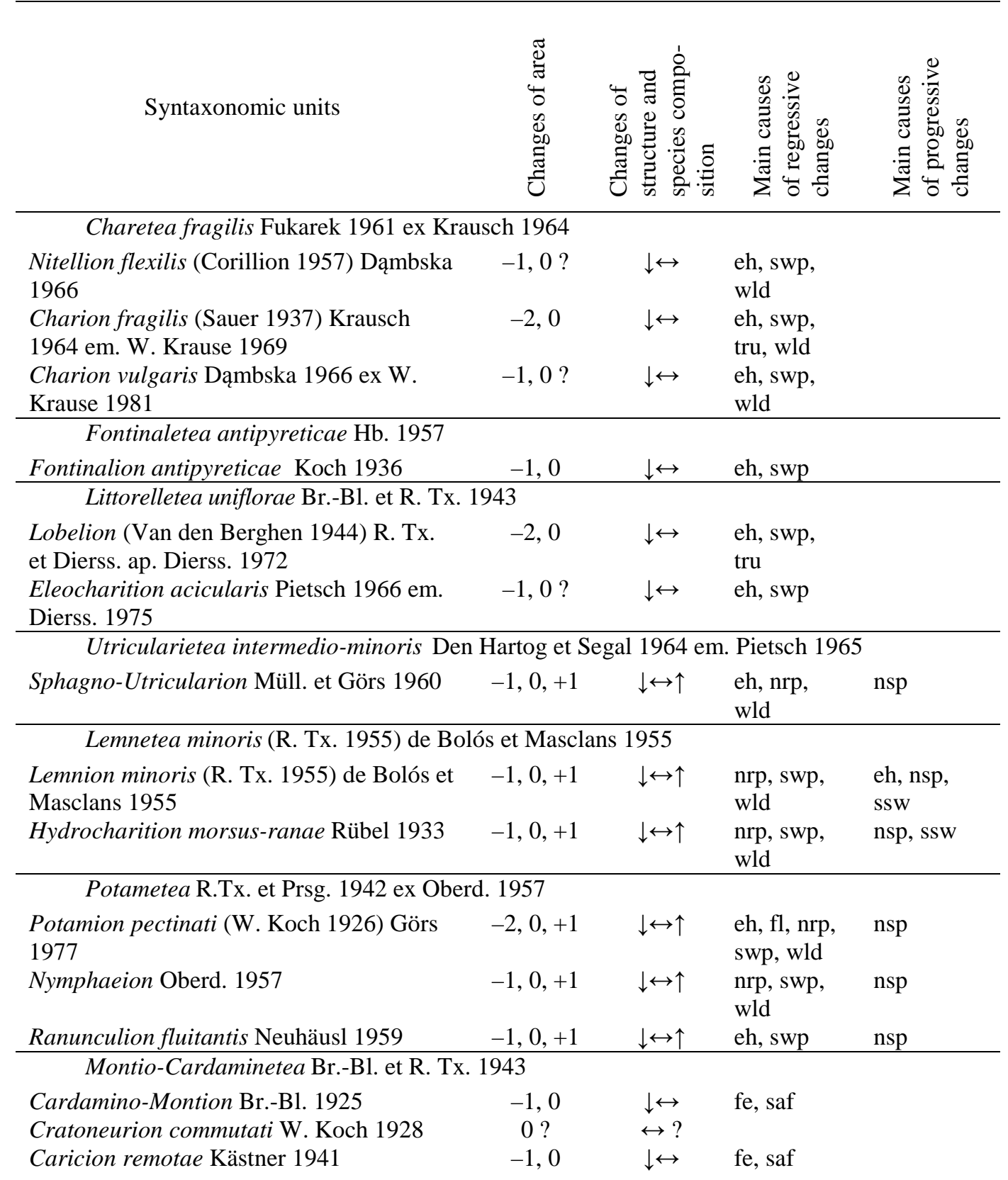


Table 4. (Continued)

\begin{tabular}{|c|c|c|c|c|}
\hline \multicolumn{5}{|c|}{ Phragmitetea australis (Klika in Klika Novák 1941) R. Tx. et Prsg 1942} \\
\hline Phragmition australis W. Koch 1926 & $-1,0,+2$ & $\downarrow \leftrightarrow \uparrow$ & $\begin{array}{l}\text { del, fe, } \\
\text { gwld, nrp, } \\
\text { swp, tbulb, } \\
\text { tru, we }\end{array}$ & $\begin{array}{l}\text { eh, nsp, } \\
\text { ssuccu, } \\
\text { ssw }\end{array}$ \\
\hline Magnocaricion elatae W. Koch 1926 & $-1,0,+2$ & $\downarrow \leftrightarrow \uparrow$ & $\begin{array}{l}\text { fe, gwld, } \\
\text { swp, trurb, } \\
\text { tru, we }\end{array}$ & nsp, ssw \\
\hline $\begin{array}{l}\text { Oenanthion aquaticae Hejný ex Neuhäusl } \\
1959\end{array}$ & $-1,0,+1$ & $\downarrow \leftrightarrow \uparrow$ & $\begin{array}{l}\text { eh, gwld, } \\
\text { swp }\end{array}$ & nsp, ssw \\
\hline $\begin{array}{l}\text { Sparganio-Glycerion fluitantis Br.-Bl. et } \\
\text { Siss. ap. Boer } 1942 \text { n.inv. }\end{array}$ & $-1,0,+1$ & $\downarrow \leftrightarrow \uparrow$ & swp, wld & nsp \\
\hline Phalaridion Kopecký 1961 & $-1,0,+2$ & $\downarrow \leftrightarrow \uparrow$ & $\begin{array}{l}\text { gwld, swp, } \\
\text { tburb }\end{array}$ & $\begin{array}{l}\text { eh, fl, nsp, } \\
\text { ssuccu, } \\
\text { ssw }\end{array}$ \\
\hline \multicolumn{5}{|c|}{ Thero-Salicornietea Pign. 1953 em. R. Tx. 1954 in R. Tx. et Oberd. 1958} \\
\hline Salicornion ramosissimae R. Tx. 1974 & $-1,0,+1$ & $\downarrow \leftrightarrow \uparrow$ & gwld, lssw & nsp \\
\hline \multicolumn{5}{|c|}{ Isoëto durieui-Juncetea bufonii (Br.-Bl. et R. Tx. 1943 ex Westh. et al. 1946) Riv.-Mart. 1988} \\
\hline $\begin{array}{l}\text { Elatino-Eleocharition ovatae Pietsch et } \\
\text { Müller-Stoll } 1968\end{array}$ & $-2,+1$ & $\downarrow \uparrow$ & $\begin{array}{l}\text { gwld, del, } \\
\text { tburb, tu }\end{array}$ & wld \\
\hline $\begin{array}{l}\text { Nanocyperion flavescentis W. Koch } 1926 \\
\text { ex Aichinger } 1933 \text { em. Rivas Goday } 1961\end{array}$ & $-1,+1$ & $\downarrow \uparrow$ & $\begin{array}{l}\text { gwld, del, } \\
\text { tbulb, tu }\end{array}$ & wld \\
\hline $\begin{array}{l}\text { Radiolion linoidis (Rivas-Goday 1961) } \\
\text { Pietsch } 1973\end{array}$ & $-2,+1$ & $\downarrow \uparrow$ & $\begin{array}{l}\text { gwld, lr, } \\
\text { tu, we }\end{array}$ & $\operatorname{tr}$ \\
\hline \multicolumn{5}{|c|}{ Bidentetea tripartitae R. Tx., Lohm. et Prsg in R. Tx. 1950} \\
\hline $\begin{array}{l}\text { Bidention tripartitae Nordh. } 1940 \text { em. R. } \\
\text { Tx. in Poli et J. Tx. } 1960\end{array}$ & $-1,+1$ & $\downarrow \uparrow$ & $\begin{array}{l}\text { asexp, del, } \\
\text { lr, tburb, } \\
\text { tu, we }\end{array}$ & nsp, ssw, tr \\
\hline $\begin{array}{l}\text { Chenopodion glauci (R. Tx.1960 in Poli et } \\
\text { J. Tx. 1960) Hejný } 1974\end{array}$ & $-2,+2$ & $\downarrow \uparrow$ & $\begin{array}{l}\text { asexp, del, } \\
\text { tburb, we }\end{array}$ & fl, nsp \\
\hline \multicolumn{5}{|c|}{ Scheuchzerio-Caricetea nigrae (Nordh. 1936) R. Tx. 1937} \\
\hline $\begin{array}{l}\text { Rhynchosporion albae W. Koch } 1926 \\
\text { Caricion lasiocarpae Vanden Bergh. in } \\
\text { Lebrun et all. } 1949\end{array}$ & $\begin{array}{c}-1,0 \\
-1,0,+1\end{array}$ & $\begin{aligned} \downarrow & \leftrightarrow \\
\downarrow \downarrow & \leftrightarrow \uparrow\end{aligned}$ & $\begin{array}{l}\text { nrp } \\
\text { aff, eh, } \\
\text { gwld, nrp }\end{array}$ & nsp, ssw \\
\hline $\begin{array}{l}\text { Caricion nigrae W. Koch } 1926 \text { em. Klika } \\
1934\end{array}$ & $-1,0,+1$ & $\downarrow \leftrightarrow \uparrow$ & $\begin{array}{l}\text { aff, eh, } \\
\text { gwld, nrp }\end{array}$ & ssw \\
\hline Caricion davallianae Klika 1934 & $-1,0,+1$ & $\downarrow \leftrightarrow \uparrow$ & $\begin{array}{l}\text { aff, gwld, } \\
\text { nrp }\end{array}$ & nsp, ssw \\
\hline \multicolumn{5}{|c|}{ Oxycocco-Sphagnetea Br.-Bl. et R. Tx. 1943} \\
\hline $\begin{array}{l}\text { Sphagnion magellanici Kästner et Flössner } \\
1933 \text { em. Dierss. } 1975\end{array}$ & $-1,0,+1$ & $\downarrow \leftrightarrow \uparrow$ & gwld, nrp & nsp \\
\hline
\end{tabular}

Semi-natural meadows, tall herbs, grasslands and forest edges vegetation (Tab. 5) is dependent both on natural and anthropogenic factors. Wet meadows (all. Molinion, Cnidion) undergo transformations as a result of use intensification or cessation thereof. Fresh pastures (all. Cynosurion), halophyte grasslands (all. Puccinellion maritimae, Armerion maritimae) and xerothermic grasslands (all. Cirsio-Brachypodion, Festuco-Stipion) are decreasing their share in consequence of 
grazing cessation. Poor grasslands and heaths (all. Violion caninae, CallunoArctostaphylion) are disappearing as a result of eutrophication, shadowing or building-up. Progressive tendencies, in turn, reveal tall herbs and nitrophilous edge communities (all. Filipendulion, Calystegion sepium, Aegopodion podagrariae, Galio-Alliarion) and fertile fresh meadows (all. Alopecurion pratensis, Arrhenatherion elatioris). Particularly significant for the last 20 years period is a secondary tall herbs succession in conditions of wet meadows' cessation of use (cf. KOCHANOWSKA 1997; KRASICKA-KORCZYŃSKA et al. 2008).

From group of ruderal and segetal vegetation (Tab. 6) regressive tendencies have very rare phytocoenoses of Caucalidion lappulae alliance, whereas progressive tendencies have meso- and thermophilic ruderal communities (all. ConvolvuloAgropyrion repentis, Onopordion acanthii, Sisymbrion, Eragrostion).

Table 5. Transformations of meadow, grassland and forest edge vegetation. Changes of area: see explanations in Table 1. Changes of structure and species composition: see explanations in Table 1. Main causes: aff - afforestation; asexp - alien species expansion; bua - built-up areas; cfag - conversion into farmlands or allotment gardens; cfu - cessation of farmlands use; eg - establishment of greenway; eh eutrophication of habitats; ff - forest felling; fl - floods; gcp - green crops ploughing; ges - grass extra sowing; gi - grazing intensification; gl - grazing limitation; gwld ground water level decrease; le - lawn establishment; lr - levelling of roadsides; lssw - limitation of supply with saline water; mi - mowing intensification; ml - mowing limitation; nrp - natural regression processes; nsp - natural succession processes; o overfertilization; $\mathrm{p}$ - plantings; saf - shadowing by adjacent forests; sge - sand and gravel exploitation; ssuccu - secondary succession due to cessation of use; ssw secondary swamping; tburb - technical building up of river banks; tr - trampling; tru - tourist and recreation use; we - wastelands elimination.

\begin{tabular}{|c|c|c|c|c|}
\hline Syntaxonomic units & 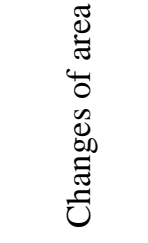 & 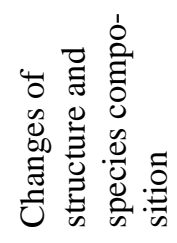 & 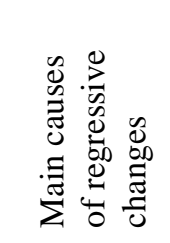 & 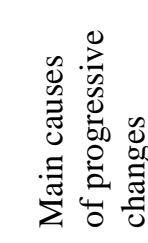 \\
\hline \multicolumn{5}{|c|}{ Molinio-Arrhenatheretea R. Tx. 1937 em. 1970} \\
\hline Lolio-Plantaginion majoris R. Tx. 1947 & $-1,0,+1$ & $\downarrow \leftrightarrow \uparrow$ & $\begin{array}{l}\text { eg, le, lr, } \\
\text { we }\end{array}$ & gi, tr, tru \\
\hline $\begin{array}{l}\text { Agropyro-Rumicion crispi Nordh. } 1940 \\
\text { em. R. Tx. } 1950\end{array}$ & $-1,0,+1$ & $\downarrow \leftrightarrow \uparrow$ & $\begin{array}{l}\text { gwld, nrp, } \\
\text { we }\end{array}$ & gi, ssw \\
\hline Filipendulion ulmariae Segal 1966 & $-1,0,+2$ & $\downarrow \leftrightarrow \uparrow \uparrow$ & $\begin{array}{l}\text { aff, gcp, } \\
\mathrm{mi}\end{array}$ & ssuccu \\
\hline Molinion caeruleae W. Koch 1926 & $-2,0,+1$ & $\downarrow \downarrow \leftrightarrow \uparrow$ & $\begin{array}{l}\text { aff, cfag, } \\
\text { eh, gcp, }\end{array}$ & gwld, ml \\
\hline
\end{tabular}


Table 5. (Continued)

\begin{tabular}{|c|c|c|c|c|}
\hline \\
\hline $\begin{array}{l}\text { Calthion palustris R. Tx. } 1936 \text { em. Oberd. } \\
1957\end{array}$ & $-1,0,+1$ & $\downarrow \downarrow \leftrightarrow \uparrow$ & $\begin{array}{l}\text { ssuccu } \\
\text { aff, gcp, } \\
\text { ges, gwld, } \\
\text { o, ssuccu }\end{array}$ & ssw, ml \\
\hline Cnidion dubii Bal.-Tul. 1966 & $-1,0$ & $\downarrow \downarrow \leftrightarrow$ & $\begin{array}{l}\text { gwld, mi, } \\
\text { o, ssuccu, } \\
\text { ssw }\end{array}$ & $\mathrm{fl}, \mathrm{ml}$ \\
\hline Alopecurion pratensis Pass. 1964 & $-1,0,+2$ & $\downarrow \leftrightarrow \uparrow$ & $\begin{array}{l}\text { asexp, aff, } \\
\text { bua, cfag, } \\
\text { gcp, ges, } \\
\text { ssuccu }\end{array}$ & eh, fl, mi \\
\hline $\begin{array}{l}\text { Arrhenatherion elatioris (Br.-Bl. 1925) } \\
\text { Koch } 1926\end{array}$ & $-1,0,+2$ & $\downarrow \downarrow \leftrightarrow \uparrow$ & $\begin{array}{l}\text { aff, bua, } \\
\text { cfag, gcp, } \\
\text { ges, gi, lr, } \\
\text { o, ssuccu }\end{array}$ & $\begin{array}{l}\mathrm{gl} \text {, gwld, } \\
\mathrm{mi}\end{array}$ \\
\hline Cynosurion R. Tx. 1947 & $-2,0,+1$ & $\downarrow \downarrow \leftrightarrow \uparrow$ & $\begin{array}{l}\text { bua, cfag, } \\
\text { ges, gl, mi, } \\
\text { ssuccu }\end{array}$ & gi \\
\hline
\end{tabular}

\begin{tabular}{|c|c|c|c|c|}
\hline \multicolumn{5}{|c|}{ Asteretea tripolium Westh. at Beeft. ap. Beeft 1962} \\
\hline $\begin{array}{l}\text { Puccinellion maritimae (Christ. 1927) R. } \\
\text { Tx. } 1937\end{array}$ & $-2,0,+1$ & $\downarrow \downarrow \leftrightarrow \uparrow$ & $\begin{array}{l}\text { gl, gwld, } \\
\text { lssw, } \\
\text { ssuccu }\end{array}$ & gi, ssw \\
\hline $\begin{array}{l}\text { Armerion maritimae Br.-Bl. et De Leeuw } \\
1936\end{array}$ & $-2,0,+1$ & $\downarrow \downarrow \leftrightarrow \uparrow$ & $\begin{array}{l}\text { gl, gwld, } \\
\text { lssw, } \\
\text { ssuccu }\end{array}$ & gi, ssw \\
\hline \multicolumn{5}{|c|}{ Koelerio glaucae-Corynephoretea canescentis Klika in Klika et Novák 1941} \\
\hline Corynephorion canescentis Klika 1934 & $-1,0,+1$ & $\downarrow \leftrightarrow \uparrow$ & $\begin{array}{l}\text { aff, bua, } \\
\text { nrp, saf, } \\
\text { sge, we }\end{array}$ & $\mathrm{cfu}, \mathrm{nsp}$ \\
\hline $\begin{array}{l}\text { Thero-Airion R. Tx. } 1951 \text { ex Oberd. } 1957 \\
\text { em. Brzeg in Brzeg et M. Wojt. } 1996\end{array}$ & $-1,0,+1 ?$ & $\downarrow \leftrightarrow \uparrow ?$ & $\begin{array}{l}\text { aff, bua, } \\
\text { cfag, nrp, } \\
\text { saf, sge, } \\
\text { we }\end{array}$ & $\mathrm{cfu}, \mathrm{nsp}$ \\
\hline Koelerion glaucae (Volk 1931) Klika 1934 & $-1,0,+1$ & $\downarrow \leftrightarrow \uparrow$ & $\begin{array}{l}\text { aff, bua, } \\
\text { cfag, nrp, } \\
\text { saf, sge, } \\
\text { we }\end{array}$ & $\mathrm{cfu}, \mathrm{nsp}$ \\
\hline
\end{tabular}

Festuco-Brometea Br.-Bl. et R. Tx. 1943

Cirsio pannonici-Brachypodion pinnati

$-2,0,+1 \quad \downarrow \downarrow \leftrightarrow \uparrow$

aff, bua, gi, nsp

Hadač et Klika in Klika et Hadač 1944 em.

Krausch 1961

Festuco-Stipion (Klika 1931) Krausch

1961

$-2,0,+1 \quad \downarrow \downarrow \leftrightarrow \uparrow$

npr,

ssuccu, we

Phleion boehmeri Głowacki 1972 ex

Celiński et Balcerkiewicz 1973

aff, bua, gi, nsp

lr, npr,

aff, bua, cfu, nsp

cfag, lr,

saf, sge,

we

Alysso alyssoidis-Sedion albi Oberd. et Th. $\quad-1,0,+1 ? \quad \downarrow \leftrightarrow \uparrow ? \quad$ lr, p, sge, $\quad$ nsp

Müll. in Th. Müll. 1961 we

Trifolio-Geranietea sanguinei Th. Müll. 1962

$\begin{array}{llll}\text { Geranion sanguinei } \mathrm{R} \text {. Tx. in Th. Müll. } & -1,0,+1 & \downarrow \leftrightarrow \uparrow & \begin{array}{l}\text { lr, nrp, p, } \\ \text { saf }\end{array}\end{array}$ 
Table 5. (Continued)

Trifolion medii Th. Müll. 1962

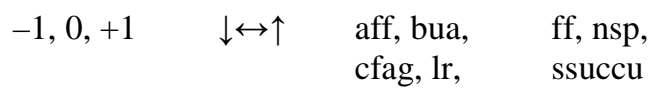

nrp, p, saf,

we

Melampyrion pratensis Pass. 1967

$$
-1,0,+1 \quad \downarrow \leftrightarrow \uparrow \quad l r, \operatorname{nrp}, \mathrm{p},
$$

ff, nsp

Nardo-Callunetea Prsg 1949

Violion caninae Schwick. 1944

$-2,0 \quad \downarrow \downarrow \leftrightarrow \quad$ aff, bua,
eh, gi, o,

Calluno-Arctostaphylion R. Tx. et Prsg

$-2,0 \quad \downarrow \downarrow \leftrightarrow$

ssuccu, we

1949 ex Faliński 1965

aff, bua,

cfag, eh,

saf, we

Pohlio nutantis-Callunion (Shimwell 1973) Brzeg 1982

$-1,0,+1 \quad \downarrow \leftrightarrow \uparrow \quad$ aff, nrp,

ff, nsp saf

\section{Asplenietea trichomanis (Br.-Bl. in Meier et Br.-Bl. 1934) Oberd. in Oberd. 1977}

Cymbalario-Asplenion Segal $1969 \mathrm{em}$.

Mucina 1993

Hypno-Polypodion vulgaris Mucina 1993 Artemisietea vulgaris Lohm., Prsg et R. Tx. in R. Tx. 1950

Calystegion sepium R. Tx. 1947 em. 1950 1947

$$
-1,0,+2 \quad \downarrow \leftrightarrow \uparrow \uparrow
$$

asexp, 1 , $\mathrm{ml}, \mathrm{nsp}$,

$\begin{array}{llll}-1,0,+2 & \downarrow \leftrightarrow \uparrow & \begin{array}{l}\text { aff, asexp, } \\ \text { lr, nrp, saf }\end{array} & \text { ssuccu } \\ -1,0,+2 & \downarrow \leftrightarrow \uparrow & \begin{array}{l}\text { asexp, lr, } \\ \text { p, saf }\end{array} & \text { ff }\end{array}$

Galio-Alliarion (Oberd. 1962) Lohm. et Oberd. in Oberd. et al. 1967 $\mathrm{p}$, saf 
Table 6. Transformations of ruderal and segetal vegetation. Changes of area: explanations in Table 1. Changes of structure and species composition: explanations in Table 1. Main causes: acu - arable cultivation; aff - afforestation; asexp - alien species expansion; bua - built-up areas; cfu - cessation of farmlands use; eh eutrophication of habitats; ha - herbicide application; le - lawn establishment; lr levelling of roadsides; o - overfertilization; p - plantings; ssuccu - secondary succession due to cessation of use; tr - trampling; tru - tourist and recreation use; we - wastelands elimination; wf - wastelands formation.

\begin{tabular}{|c|c|c|c|c|}
\hline Syntaxonomic units & 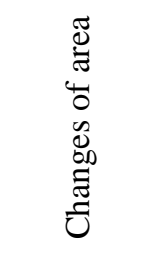 &  & 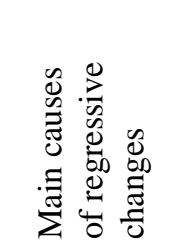 & 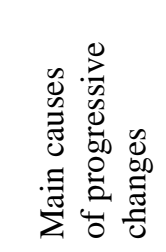 \\
\hline \multicolumn{5}{|c|}{ Polygono arenastri-Pö̈tea annuae Riv.-Mart. 1975 corr. Riv.-Mart. et al. 1991} \\
\hline $\begin{array}{l}\text { Matricario matricarioidis-Polygonion } \\
\text { arenastri Riv.-Mart. } 1975 \text { corr. Riv.-Mart. } \\
\text { et al. } 1991\end{array}$ & $-1,0,+1$ & $\downarrow \leftrightarrow \uparrow$ & $\begin{array}{l}\text { bua, eg, le, } \\
\text { lr, we }\end{array}$ & $\operatorname{tr}, \operatorname{tru}$ \\
\hline $\begin{array}{l}\text { Saginion procumbentis R. Tx. et Ohba } \\
1972 \text { in Géhu et al. } 1972\end{array}$ & $-1,0,+1$ & $\downarrow \leftrightarrow \uparrow$ & $\begin{array}{l}\text { bua, eg, le, } \\
\text { lr, we }\end{array}$ & tr, tru \\
\hline \multicolumn{5}{|c|}{ Agropyretea intermedio-repentis (Oberd. et all. 1967) Th. Müll. et Görs 1969} \\
\hline $\begin{array}{l}\text { Convolvulo arvensis-Agropyrion repentis } \\
\text { Görs } 1966\end{array}$ & $-1,0,+2$ & $\downarrow \leftrightarrow \uparrow$ & $\begin{array}{l}\text { asexp, aff, } \\
\text { bua, eg, le, } \\
\text { lr, p, we }\end{array}$ & $\begin{array}{l}\text { eh, cfu, } \\
\text { ssuccu }\end{array}$ \\
\hline \multicolumn{5}{|c|}{ Artemisietea vulgaris Lohm., Prsg et R. Tx. in R. Tx. 1950} \\
\hline $\begin{array}{l}\text { Onopordion acanthii Br.-Bl. } 1926 \text { ex Br.-Bl. } \\
\text { et al. } 1936\end{array}$ & $-1,0,+2$ & $\downarrow \leftrightarrow \uparrow$ & $\begin{array}{l}\text { asexp, aff, } \\
\text { bua, eg, le, } \\
\text { lr, p, we }\end{array}$ & $\mathrm{cfu}, \mathrm{eh}, \mathrm{wf}$ \\
\hline $\begin{array}{l}\text { Arction lappae R. Tx. } 1937 \text { em. Siss. in } \\
\text { Westh. et al. } 1946\end{array}$ & $-1,0,+1$ & $\downarrow \leftrightarrow \uparrow$ & $\begin{array}{l}\text { bua, eg, le, } \\
\text { lr, p, we }\end{array}$ & eh, wf \\
\hline \multicolumn{5}{|c|}{ Stellarietea mediae R. Tx., Lohm. et Prsg in R. Tx. 1950} \\
\hline Panico-Setarion Siss. in Westh. et al. 1946 & $-1,0,+1$ & $\downarrow \leftrightarrow \uparrow$ & ha, o & acu \\
\hline $\begin{array}{l}\text { Scleranthion annui (Kruseman et Vlieger } \\
\text { 1939) Siss. in Westh. et al. } 1946\end{array}$ & $-1,0,+1$ & $\downarrow \downarrow \leftrightarrow \uparrow$ & $\begin{array}{l}\text { asexp, ha, } \\
\text { o }\end{array}$ & $\mathrm{acu}$ \\
\hline $\begin{array}{l}\text { Veronico-Euphorbion Siss. } 1942 \text { ex Pass. } \\
1964\end{array}$ & $-1,0,+1$ & $\downarrow \leftrightarrow \uparrow$ & $\begin{array}{l}\text { asexp, ha, } \\
\text { o }\end{array}$ & $\mathrm{acu}$ \\
\hline Caucalidion lappulae R. Tx.1950 & $-1,0$ & $\downarrow \leftrightarrow$ & ha, o & $\mathrm{acu}$ \\
\hline $\begin{array}{l}\text { Sisymbrion R. Tx., Lohm. et Prsg in R. Tx. } \\
1950\end{array}$ & $-1,0,+2$ & $\downarrow \leftrightarrow \uparrow$ & $\begin{array}{l}\text { asexp, eg, } \\
\text { le, lr, we }\end{array}$ & eh, wf \\
\hline $\begin{array}{l}\text { Malvion neglectae (Gutte 1966) Hejný } \\
1978\end{array}$ & $-1,0,+1$ & $\downarrow \leftrightarrow \uparrow$ & $\begin{array}{l}\text { eg, le, lr, } \\
\text { we }\end{array}$ & eh, wf \\
\hline Eragrostion R. Tx. in Slavnić 1944 & $-1,0,+2$ & $\downarrow \leftrightarrow \uparrow$ & $\begin{array}{l}\text { asexp, lr, } \\
\text { we }\end{array}$ & eh, wf \\
\hline
\end{tabular}




\section{CONCLUSIONS}

- Acreage changes and transformations of different vegetation types of examined region in a period of the last 20 years are both effects of factors existing mainly only in analyzed time period. Into first group regressive changes of wet riparian poplar and elm forests (Populetum albae, Ficario-Ulmetum minoris) and gradual overgrowing of xerothermic grasslands of Festuco-Brometea class can be reckoned. Whereas to the second group belong i.a. changes resulting from cessation of use of meadows and pastures and frequent mixed coniferous forests regeneration on account of proecological forest management.

- Frequent and drastic plant cover changes of swamp ecosystems as a result of anthropogenic ground water level lowering, which occurred in the middle of the 20th century, are not observed currently. Nowadays even often an overgrowing of drainage ditches and secondary swamping takes place, which is confirmed by progressive dynamic tendencies of rush vegetation. Preserved natural moss bogs undergo gradual overgrowing so they do not belong to strongly endangered plant cover elements of examined area. Also less often an intensive meadow management in grasslands and farmlands is applied. Moreover, greater preferences in forest management have deciduous species than introduced until recently on a large scale pine Pinus sylvestris.

- Shown regressive and progressive changes of different vegetation types are, to a larger degree (than in the middle of the $20^{\text {th }}$ century) generated by natural factors rather than anthropogenic factors. Disappearing and transformation of thermophilous oak forests, dry and fresh coniferous forests, halophytic, pasture, meadow, heathery and grassland vegetations result currently most of all from natural

- regeneration processes and secondary succession. At the same time, these factors create progressive changes in case of mixed coniferous forests, mesophytic shrubs, rush and tall herbs vegetation as well as fresh meadows vegetation. 


\section{REFERENCES}

BARABASZ, B. 1997. Zmiany roślinności łąk w północnej części Puszczy Niepołomickiej w ciągu 20 lat. Studia Nat., Ser. A 43: 1-99.

Berndt, J., Ceynowa-GieŁdon, M. 1988. Zmiany w runie rezerwatu Cisy Staropolskie im. L. Wyczółkowskiego (Wierzchlas) w latach 1953-1982. Ochr. Przyr. 46: 9-34.

BiAŁY, K., ZAŁuski, T. 1994. Rola bobra europejskiego Castor fiber L. w renaturyzacji uregulowanego cieku i przyległego otoczenia. Zesz. Nauk. AR we Wrocławiu 246, Konferencje 3 (1): 21-29.

BodziARCZyK, J., Drajewicz, R. 2007. Dynamika roślinności na opuszczonych polanach Pienińskiego Parku Narodowego. In: J. HoleKSA (ed.), Zakres, tempo i mechanizmy zmian w przyrodzie terenów chronionych w Polsce. Studia Nat. 54, cz. I: $13-46$.

BRZEG, A., WoJTERsKA, M. 2001. Zespoły roślinne Wielkopolski, ich stan poznania i zagrożenie. In: M. WoJTERSKA (ed.), Szata roślinna Wielkopolski i Pojezierza Południowopomorskiego. Przewodnik sesji terenowych 52. Zjazdu Polskiego Towarzystwa Botanicznego 24-28 września 2001. Bogucki Wydawnictwo Naukowe, Poznań, pp. 39-110.

CyzMan, W. 1991. mscr. Zróżnicowanie zbiorowisk leśnych i zaroślowych w Kotlinie Toruńskiej i Kotlinie Płockiej. Zakład Taksonomii i Geografii Roślin UMK, Toruń.

CYZMAN, W. 2009. Zasięg i natężenie zmian w zespołach leśnych rezerwatu „Olszyny Rakutowskie” w latach 1969-2002. In: K. KANNENBERG, H. SzRAMKA (eds), Zarządzanie ochroną przyrody w lasach. Tom III. Wyd. Wyższej Szkoły Zarządzania Środowiskiem w Tucholi, Tuchola, pp. 33-62.

Cyzman, W., Rejewski, M. 1992. Przekształcenia zespołów leśnych w okolicach Jeziora Rakutowskiego w latach 1969-1988. Acta Univ. Nic. Copern., Biologia 40: $121-136$.

Cyzman, W., Kannenberg, A. 2008. Zmiany w składzie florystycznym i strukturze zbiorowisk leśnych w wybranych rezerwatach na Kujawach i Pomorzu. In: K. KANNENBERG, H. SzRAMKA (eds), Zarządzanie ochroną przyrody w lasach. Tom 
II. Wyd. Wyższej Szkoły Zarządzania Środowiskiem w Tucholi, Tuchola, pp. 91-108.

DURAK, T. 2009. Changes in rich fir-beech forests in the Beskid Niski Mountains during 30 years. In: J. HoleKsa, B. BABCZYŃSKA-SENDEK, S. WiKa (eds), The role of geobotany in biodiversity conservation. University of Silesia, Katowice, pp. 141-148.

EJANKOWSKI, W., KUNZ, M. 2006. Reconstruction of vegetation dynamics in "Linje" peat-bog ( $\mathrm{N}$ Poland) using remote sensing method. Biodiv. Res. Conserv. 1-2: 111-113.

FALIŃSKA, K. 2004. Ekologia roślin. Wyd. 3. Wydawnictwo Naukowe PWN, Warszawa.

GryniA, M., KrysZAK, A., KrysZAK, J. 2009. Transformations of meadows communities in the Obra River valley. In: J. HOLEKSA, B. BABCZYŃSKA-SENDEK, S. WIKA (eds), The role of geobotany in biodiversity conservation. University of Silesia, Katowice, pp. 149-153.

Herbichowa, M., WoŁejKo, L. 2004. Torfowiska źródliskowe i przepływowe Polski północnej. In: J. HERBICH (ed.), Poradniki ochrony siedlisk i gatunków Natura 2000 - podręcznik metodyczny. Tom 2. Wody słodkie i torfowiska. Ministerstwo Środowiska, Warszawa, pp. 191-195.

HINTERLANG, D. 1992. Vegetationsökologie der Weichwasserquellgesellschaften zentraleuropäischer Mittelgebirge. Crunoecia 1: 5-117.

Jagodziński, A. M., MaciejewskA-RutKowskA, I. 2005. Flora naczyniowa i roślinność rezerwatu „Ostrów Panieński” koło Chełmna. Parki Nar. Rez. Przyr. 24 (1-4): 61-87.

JAGODZIŃSKI, A. M., MACIEJEWSKA-RUTKOwsKA, I. 2008. Zmiany we florze rezerwatu przyrody „Ostrów Panieński” koło Chełmna. In: J. HolEKsA (ed.), Zakres, tempo i mechanizmy zmian w przyrodzie terenów chronionych w Polsce. Studia Nat. 54, cz. II: 121-131.

JAKUBOWSKA-GABARA, J. 1993. Recesja zespołu świetlistej dąbrowy Potentillo albae-Quercetum Libb. 1993 w Polsce. Wydawnictwo Uniwersytetu Łódzkiego, Łódź. 
JutrZenkA-Trzebiatowski, A. 1999. Wpływ człowieka na szatę leśną Polski północno-wschodniej w ciągu dziejów. Rozprawy i Materiały Ośrodka Badań Naukowych im. W. Kętrzyńskiego w Olsztynie 184: 1-172.

KAŹMIERCZAKOWA, R., GRodZIŃSKA, K. 2007. Przemiany zbiorowisk naskalnych i kserotermicznych w Pienińskim Parku Narodowym w ciągu ostatnich 35 lat XX wieku. In: J. HoLEKSA (ed.), Zakres, tempo i mechanizmy zmian w przyrodzie terenów chronionych w Polsce. Studia Nat. 54, cz. I: 85-132.

KęPCZYŃSKI，K., CEYNOWA-GIEŁDON, M. 1988. Roślinność brzeżna Zalewu Koronowskiego w okresie obniżonego poziomu wody w zbiorniku. Acta Univ. Nic. Copern., Biologia 29: 25-51.

KĘPCZYŃSKI, K., ZAŁUSKI, T. 1977. Obserwacje nad zmianami szaty roślinnej w rejonie oddziaływania Zakładów Azotowych "Włocławek". Acta Univ. Nic. Copern., Biologia 20: 113-125.

KĘPCZYŃSKI, K., ZAŁUSKI, T. 1988. Przekształcenia wybranych fitocenoz szuwarowych, olsowych, łęgowych i ruderalnych pod wpływem oddziaływania Zakładów Azotowych "Włocławek". Acta Univ. Nic. Copern., Biologia 32: 105118.

KĘPCZYŃSKI, K., ZAŁUSKI, T. 1991. Przekształcenia fitocenoz borów sosnowych i mieszanych w pobliżu Zakładów Azotowych "Włocławek". Acta Univ. Nic. Copern., Biologia 38: 3-22.

KĘPCZYŃSKI, K., ZIELSKI, A. 1976. Zmiany w runie zbiorowisk leśnych Pojezierza Brodnickiego pod wpływem turystyki. Phytocoenosis 5 (3/4): 387-396.

KochanOwSKA, R. 1997. Przyrodnicze konsekwencje regresu gospodarki łąkowej na Pomorzu Zachodnim. Przegl. Przyr. 8 (1-2): 73-76.

KONDRACKI, J. 2000. Geografia regionalna Polski. Wyd. 2. Wydawnictwo Naukowe PWN, Warszawa.

KoRNAŚ, J. 1987. Zmiany roślinności segetalnej w Gorcach w ostatnich 35 latach. Zesz. Nauk. Uniwersytetu Jagiellońskiego, Pr. Bot. 15: 7-26.

KoRnAŚ, J., DubIEL, E. 1991. Changes of vegetation of the hay-meadows in the Ojców National Park (S. Poland) during the last 30 years. In: J. B. FALIŃSKI (ed.), Vegetation processes as subject of geobotanical maps. Proceedings of XXXIII 
Symposium of IAVS, Warsaw, April 8-12, 1990. Phytocoenosis 3 (N.S.), Suppl. Cartogr. Geobot. 2, pp. 135-144.

KrASICKA-KoRCZYŃSKA, E. 1996. Regeneracja populacji Senecio paluster (L.) DC. na spalonych łąkach torfowiskowych w dolinie Noteci, czyli krótkie panowanie. Przegl. Przyr. 7 (3-4): 139-144.

KrasickA-KorczyńsKa, E., ZAŁuski, T., RAtyŃsKa, H., KorCZyŃsKi, M. 2008. Roślinność siedlisk łąkowych i użytków przyrodniczych w regionie kujawskopomorskim. Podręcznik dla doradców rolnośrodowiskowych. KujawskoPomorski Ośrodek Doradztwa Rolniczego w Minikowie, Minikowo.

KUCHARSKI, L. 1999. Szata roślinna łąk Polski Środkowej i jej zmiany w XX stuleciu. Wydawnictwo Uniwersytetu Łódzkiego, Łódź.

KunZ, M., Nienartowicz, A. 2010. Temporal and spatial changes in the distribution of heaths within the city of Torun according to analysis of cartographic and remote-sensing materials, and field exploration. Ecol. Questions 12: 59-74.

KUROWSKI, J. K. 1993. Dynamika fitocenoz leśnych w rejonie kopalni odkrywkowej Bełchatów. Wydawnictwo Uniwersytetu Łódzkiego, Łódź.

KuROwSKI, J. K., MichalSKA-HEJDUK, D. 2007. Tendencje dynamiczne zbiorowisk nieleśnych Kampinoskiego Parku Narodowego. In: J. HolEKSA (ed.), Zakres, tempo i mechanizmy zmian w przyrodzie terenów chronionych w Polsce. Część I. Studia Nat. 54, cz. I: 145-157.

MARCYSIAK, K. 1999. Zbiorowiska zaroślowe jako przejaw dynamiki roślinności na przykładzie Leśnego Parku Kultury i Wypoczynku w Bydgoszczy. Przegl. Przyr. $10(3-4): 121-127$.

MATUSZKIEWICZ, J.M. (ed.) 2007a. Geobotaniczne rozpoznanie tendencji rozwojowych zbiorowisk leśnych w wybranych regionach Polski. Polska Akademia Nauk, Instytut Geografii i Przestrzennego Zagospodarowania im. Stanisława Leszczyckiego, Monografie 8, pp. 1-976.

MATUSZKIEwICZ, J.M. 2007b. Przemiany borów suchych i świeżych w zachodniej części Borów Tucholskich. In: J.M. MATUSZKIEwICZ (ed.), Geobotaniczne rozpoznanie tendencji rozwojowych zbiorowisk leśnych w wybranych regionach Polski. IGiPZ PAN, Monografie 8, pp. 96-116. 
MATUSZKIEwiCZ, J.M. 2007c. Ogólne kierunki zmian w zbiorowiskach leśnych Polski, ich przyczyny oraz prognoza przyszłych kierunków rozwojowych. In: J. M. MatuszKiEwiCZ (ed.), Geobotaniczne rozpoznanie tendencji rozwojowych zbiorowisk leśnych w wybranych regionach Polski. IGiPZ PAN, Monografie 8, pp. 555-816.

MAtuszKiewiCZ, W. 2005. Przewodnik do oznaczania zbiorowisk roślinnych Polski. Vademecum geobotanicum. Wydawnictwo Naukowe PWN, Warszawa.

MichALIK, S. 1974. Antropogeniczne przemiany szaty roślinnej Ojcowskiego Parku Narodowego od początków XIX wieku do 1960 roku. Ochr. Przyr. 39: 65-154.

Michalik, S. 1990a. Przemiany roślinności kserotermicznej w czasie 20-letniej sukcesji wtórnej na powierzchni badawczej „Grodzisko” w Ojcowskim Parku Narodowym. Prądnik. Pr. Muz. Szafera 2: 43-52.

MichALIK, S. 1990b. Sukcesja wtórna półnaturalnej murawy kserotermicznej Origano-Brachypodietum w latach 1960-1984 wskutek zaprzestania wypasu w rezerwacie Kajasówka. Prądnik. Pr. Muz. Szafera 2: 59-65.

MiCHALSKA-HEJDUK, D. 2007. Zmiany w składzie gatunkowym łąk trzęślicowych Molinietum caeruleae Kampinoskiego Parku Narodowego w latach 1994-2004. In: J. HoleKSA (ed.), Zakres, tempo i mechanizmy zmian w przyrodzie terenów chronionych w Polsce. Studia Nat. 54, cz. I: 159-172.

Nienartowicz, A., Kunz, M., Adamska, E., Boińska, U., DeptuŁa, M., GugNACKA-FIEDOR, W., KAMIŃSKI, D., RUTKOWSKI, L. 2010. Relief and changes in the vegetation cover and the flora of the Zadroże Dune near the city of Torun: Comparison of the conditions in 1948 and 2009. Ecol. Questions 12: 17-49.

NORYŚKIEWICZ, A. 1978. Zbiorowiska roślinne torfowiska Zgniłka oraz zmiany zachodzące w nich pod wpływem gospodarki człowieka, Stud. Soc. Scient. Torun. D, 10 (3): 3-99.

OlaCZEK R. 1976. Zmiany w szacie roślinnej Polski od połowy XIX wieku do lat bieżących. Zesz. Probl. Post. Nauk Roln. 177: 369-408.

OLACZEK, R. 1974. Etapy pinetyzacji grądu. Phytocoenosis 3 (3/4): 201-214.

PASzEK, I. (ed.) 2005. mscr. Rezerwat przyrody „Mszar Płociczno”. Plan ochrony na okres 1.01.2006-31.12.2025. VITIS Iwona Paszek, Bydgoszcz. 
RATYŃSKA, H. 2008. Bioróżnorodność użytków zielonych regionu kujawskopomorskiego. In: S. KACZMAREK (ed.), Krajobraz i bioróżnorodność. Wydawnictwo Uniwersytetu Kazimierza Wielkiego, Bydgoszcz, pp. 106-127.

REJEWSKI, M. 1981. Roślinność jezior rejonu Laski w Borach Tucholskich. Wydawnictwo Uniwersytetu Mikołaja Kopernika, Toruń.

SAMOSIEJ, L. 1987. Wpływ czynników antropogenicznych na strukturę szaty roślinnej Jeziora Chodeckiego. Acta Univ. Lodz., Folia Bot. 5: 79-107.

SENDEK, A. 1981. Analiza antropogenicznych przemian w szacie roślinnej Górnośląskiego Okręgu Przemysłowego. Pr. Nauk. Uniwersytetu Śląskiego w Katowicach 457: 1-119.

Sugier, P., CZARNECKA, B. 2004. Transformations of aquatic and mire vegetation in catchment areas of selected lakes in the Łęczna-Włodawa Lakeland. In: L. WoŁejko, J. Jasnowska (eds), The future of Polish mires. Societas Scientiarum Stetinensis, Agricultural University of Szczecin, Szczecin, pp. 137-141.

Waldon, B., RAPACKA-GaCKOWSKA, A. 2010. Stan zachowania i problemy ochrony muraw kserotermicznych w dolinach Wisły i Noteci. In: H. RATYŃSKA, B. WALdon (eds), Ciepłolubne murawy w Polsce - stan zachowania i perspektywy ochrony. Wydawnictwo Uniwersytetu Kazimierza Wielkiego, Bydgoszcz, pp. 110-127.

WiLKOŃ-MichalSKA， J. 1970. Zmiany sukcesyjne w rezerwacie halofitów „Ciechocinek” w latach 1954-1965. Ochr. Przyr. 35: 25-51.

WRÓBEL, M. 2007. Dynamika roślinności łąkowej w warunkach stosowania ciągłych zabiegów ochronnych w Pienińskim Parku Narodowym. In: J. HoleKSA (ed.), Zakres, tempo i mechanizmy zmian w przyrodzie terenów chronionych w Polsce. Studia Nat. 54, cz. I: 241-264.

ZAŁUSKI, T. 2002. Changes of vegetation in the "Bielinek" nature reserve. Ecol. Questions 2: 175-180.

ZaŁusKi, T., GaWendA, D. 1999. Antropogeniczne przekształcenia grądu TilioCarpinetum w warunkach umiarkowanych form gospodarki leśnej w Górznieńsko-Lidzbarskim Parku Krajobrazowym. Przegl. Przyr. 10 (3-4): 111116. 
ZaŁuski, T., Gawenda-Kempczyńska, D., Paszek, I., PuchaŁKa, R. 2005 mscr. Szata roślinna Łąk Bryńskich (woj. warmińsko-mazurskie) - stan zachowania, zagrożenia i projekt czynnej ochrony. Opracowanie wykonane dla GórznieńskoLidzbarskiego Parku Krajobrazowego, VITIS Iwona Paszek, Bydgoszcz.

ZaŁuski, T., Gawenda-Kempczyńska, D., PAszek, I., Rapacka-Gackowska, A. 2008. Formation of flora and vegetation on the emerging lake bed of the Rakutowskie Lake. Ecol. Questions 9: 87-92.

ZARZYCKI, J., KAŹMIERCZAKOWA, R. 2007. Przemiany łąk świeżych i pastwisk w Pienińskim Parku Narodowym w ciągu ostatnich 35 lat XX wieku. In: J. HOLEKSA (ed.), Zakres, tempo i mechanizmy zmian w przyrodzie terenów chronionych w Polsce. Studia Nat. 54, cz. I: 275-304.

ZARZYCKI, K., KorZeniaK, U. 1992. Roślinność łąkowa Pienin i jej przemiany w ostatnim sześćdziesięcioleciu. Pieniny - Przyroda i Człowiek 2: 5-12.

ZARZYCKI, K., SZELĄG, Z. 2006. Red list of the vascular plants in Poland. In: Z. MireK, K. ZARZYCKI, W. Wojewoda, Z. SzeląG (eds), Red list of plants and fungi in Poland, Czerwona lista roślin i grzybów Polski. W. Szafer Institute of Botany, Polish Academy of Sciences, Kraków, pp. 9-20.

ZIELONY, R. 1988. Form of anthropogenic degeneration of forest associations in the region of Włocławek. Ann. Warsaw Agricult. Univ.-SGGW-AR, For. and Wood Technol. 37: 63-68.

ZIELSKI, A. 1978. Zespoły leśne Pojezierza Brodnickiego oraz wpływ na nie gospodarki leśnej i turystyki. Stud. Soc. Scient. Toruń. D, 10, 4: 3-87. 